\title{
The effects of a dyadic strength-based empowerment program on the health outcomes of people with mild cognitive impairment and their family caregivers: a randomized controlled trial
}

This article was published in the following Dove Press journal:

Clinical Interventions in Aging

\section{Doris Sau-Fung Yu $\mathbb{D I D}^{\prime}$ \\ Polly Wai-chi Li (D) \\ Fan Zhang $\mathbb{D}^{2}$ \\ Sheung-Tak Cheng $\left(\mathbb{D}^{2}\right.$ \\ Tsz Kwan $\mathrm{Ng}^{3}$ \\ Katherine $\mathrm{S}$ Judge ${ }^{4}$}

'The Nethersole School of Nursing, The Chinese University of Hong Kong, Shatin, Hong Kong; ${ }^{2}$ Department of Health and Physical Education, The Education University of Hong Kong, Tai Po, Hong Kong; ${ }^{3}$ Fong Shu Chuen District Elderly Community Centre, Tung Wah Group Hospital, Sheung Wan, Hong Kong; ${ }^{4}$ Department of Psychology, College of Sciences and Health Professions, Cleveland State University, Cleveland, $\mathrm{OH}$, USA
Correspondence: Doris Sau-Fung Yu The Nethersole School of Nursing, The Chinese University of Hong Kong, Rm. 729, Esther Lee Building, Shatin, New Territories, Hong Kong

Tel +852 39434289

Fax +852 26035269

Email dyu@cuhk.edu.hk
Purpose: As an intermediate prodromal stage of dementia, mild cognitive impairment (MCI) causes functional, emotional, and social challenges for both of the person with MCI (PwMCI) and their family caregiver. However, major attention has only been placed on the PwMCI's for cognitive training. This study evaluated a more comprehensive intervention, which integrated both strength-based and empowerment approaches, to address their complex needs in a dyadic fashion.

Patients and methods: This randomized controlled trial allocated $103 \mathrm{MCI}$ patient-caregiver dyads to receive a 14-week dyadic strength-based empowerment program (D-StEP-MCI, $\mathrm{n}=52)$ or usual care $(\mathrm{n}=51)$. The $\mathrm{D}$-StEP-MCI program consisted of group-based session to the PwMCI, home-based dyadic sessions, and telephone follow-up, with the activities supported the care dyads to navigate their own strengths and resources for integration, together with the trained skills, to optimize role and social engagement in everyday life. Dyadic health outcomes in terms of subjective and objective cognitive function, and neuro-psychiatric symptoms of PwMCI, stress in symptom management of their family caregivers, and depression of the dyads were evaluated at baseline, after the D-StEP-MCI and at 3 months thereafter.

Results: By using general estimating equation, the D-StEP-MCI significantly improved the cognitive function, subjective memory, and mood status of the PwMCI, and the positive changes were maintained at the 3-month endpoints. It also has significantly positive effects on caregivers' stress in symptom management and level of depression.

Conclusion: Our findings showed the health benefit of combining strength-based and empowerment approach in supporting the disease adaptation of PwMCI and caregiver in a dyadic fashion. This study also supports the use of a social interaction approach to optimize the everyday engagement of the PwMCI.

Keywords: mild cognitive impairment, caregiving, empowerment, strength-based, dyadic approach

\section{Introduction}

In the past decades, the sharp rise in dementia cases has raised the worldwide attention of scholars, policy makers, and mass media. Approximately 46.8 million people have been affected by dementia in 2015, and the number was projected to be doubled every 20 years. ${ }^{1}$ Along with this "dementia avalanche", the prevalence of mild cognitive impairment (MCI), an intermediate prodromal stage of dementia, has also increased up 
to $20 \%$ and ranged from $5 \%$ to $37 \%$ among people aged above 60 across different populations. ${ }^{2,3}$ According to a 15year longitudinal study, roughly $12 \%$ of the persons with MCI (PwMCI) would convert to Alzheimer's disease or other type of dementia every year, and within 6 years, over $80 \%$ of MCI patients have met the diagnostic criteria of dementia. ${ }^{4}$ Considering that no reliable disease-modifying treatment has been available for dementia yet, most attention has been placed to strengthen the cognitive function of PwMCI, and hopefully delaying or even preventing the progression into dementia.

However, strengthening the cognitive function of PwMCI may not adequately address the more complex care needs of this clinical cohort. Substantial evidence has indicated that living with MCI is full of functional, emotional, and social challenges. ${ }^{5}$ The memory deficit and loss of skills in complex activities of daily living greatly compromise the self-confidence of PwMCI and cause frustration. Such reduced functional performance, together with the fear of embarrassment and the need to maintain status quo, render the PwMCI to be more withdrawn from role and social participation. The disengagement would also be reinforced when family caregivers attempt to exclude the PwMCI from family participation because of the perceived increase in responsibilities and distress in coping with their reduced functional performance. ${ }^{6}$ Indeed, the highly prevalent neuro-psychiatric symptoms particularly depression, apathy, irritability among the PwMCI also cause significant interaction difficulties and relationship strain between the PwMCI and their family caregiver, and eventually worsens the family solidarity. Over one-third of the caregivers of PwMCI have, indeed, reported clinically significant burden relating to managing the symptoms of MCI, social loss, and increased psychological distress. ${ }^{7}$

Nevertheless, the premature role and social disengagement of PwMCI are detrimental to the disease progression. According to the mental-exercise hypothesis, ${ }^{8}$ keeping mentally active would help maintain one's cognitive capacity, and even prevent possible decline and the progression of dementia, also known as "use it or lose it" adage. The reduced family solidarity and ineffective social interaction between the PwMCI and caregiver also aggravate the manifestation of neuro-psychiatric symptoms, which, in turn, increases the risk of disease progression. As such, effective strategies to manage MCI should go beyond strengthening the cognitive function of PwMCI, but to use a comprehensive approach to optimize their continuous role and social participation in an engaging family context. This is especially true, as PwMCI reserve relatively better cognitive capacities and daily functioning, thus have greater potential to be benefited from the intervention programs that navigate their remained functioning, available resources, and advance their sense of control. Using this approach may also enable family members to interact more constructively with the PwMCI and focus on their role fulfillment rather than performance inadequacy, which may in turn, benefits the caregivers' adaptation.

Up to our understanding, there is limited investigation on using a comprehensive approach to promote disease adaptation of PwMCI in a family context. Indeed, family caregivers have seldom been involved as the target of intervention, even though some studies monitored the impact of interventions administered to PwMCI on caregivers' health outcomes. ${ }^{7}$ There were only three studies administered the interventions to the PwMCI-caregiver dyads to promote the disease adaptation. Banningh et al, ${ }^{9}$ administered cognitive rehabilitation and psychoeducation to both PwMCI and their family caregivers, with content focusing on enhancing memory function, stress management, self-regulation, communication, and social conflict management. For each of the 10 bi-weekly 120-min sessions, the first 60 mins provided training to the groups of PwMCI and the caregivers separately, and the latter 30 mins was spent on the PwMCI-caregiver dyads together. However, the intervention was not effective to improve the emotional status, symptoms, well-being, sense of competence, and disease acceptance of the care dyads. The authors explained such findings as relating to the possible floor effect due to the lesser disease impact at the early stage of MCI. Another pilot study evaluated the effects of Daily Engagement of Meaningful Activities among the PwMCI and caregiver dyads. ${ }^{10}$ Problem-solving therapy was used to support the care dyads to identify and implement meaningful activities through goal achievement and problem-solving in six bi-weekly sessions. Self-management tool kit was also offered to support the coping with MCI. Again, as compared with the control group, the DEAM did not outweigh the benefits of information support in improving the emotional outcomes for the PwMCI and the caregivers. Such results may be explained by the inadequate statistical power for pilot-scale study, but may also relate to the lack of use of a comprehensive approach to tackle the life challenges of MCI. The only significant effect was found in the study by Schmitter-Edgecombe \& Dyck, $^{11}$ in which 20 twice weekly multifamily memory 
strategy training and problem-solving sessions were provided to the MCI dyads. With the trained strategies, each dyad identified goals for daily activities, developed and implemented a structured plan for goal attainment. In the post-intervention assessment, only the caregivers in the treatment group, but not the usual care group, reported a significant improvement in coping and little positive changes in depressive symptoms. However, the intervention effects on the PwMCI's outcomes were not assessed.

In the past decade, evidence has been accumulated on the dyadic intervention for person with dementia (PwD) and their caregiver ${ }^{12}$ which showed that addressing both parties' needs simultaneously could create a supportive environment for the interactions, as well as develop communication and behavioral strategies more rigorously. Built upon a strength-based concept, Judge et al, ${ }^{13}$ conducted a dyadic intervention to identify the strengths and capabilities of the PwD-caregiver dyads and integrate such performance function to enhance memory management, communication, emotional and behavioral symptom control, and social engagement. By implementing the dyadic intervention in the home setting of the care dyads, the training could also be readily translatable to their daily routine and living environment. Compared with the control intervention, the caregivers in the strength-based dyadic programs reported significantly lower caregiver burden, relationship strain, role captivity, and enhanced wellbeing. ${ }^{13}$ Yet, no evaluation of the health outcomes of the PwD was reported.

Extending the application of the strength-based dyadic approach to PwMCI would be even more appropriate, as their more preserved cognitive capacities would require a more facilitating rather than inhibiting social environment to encourage their active and self-directed role and social participation. Family caregivers become the key stakeholder who require the knowledge, skill, and attitude to create such a social context. Besides, supporting the PwMCI-caregiver dyads to better regulate the neuro-psychiatric symptoms, increase their awareness to the good qualities in the pre-morbid dyadic relationship and encourage effective use of previously established interpersonal skills are important to foster constructive dyadic relationship building. ${ }^{6,14}$ Such relational quality is considered as highly desirable to facilitate effective coping with the life challenges associated with MCI from a social interactional perspective. $^{6}$

A critical element for the success of the strength-based approach is to facilitate the clients to integrate the identified strengths to the disease adaptation process. Empowerment approach may be highly relevant to serve this purpose. This approach emphasizes on developing a partnership relationship with the clients and carefully attending to their abilities, interest, values, preferences, and psychosocial contexts in care planning. ${ }^{15}$ Collaborative effort is placed on increasing the clients' self-awareness of the need to pursue better disease adjustment, motivating the clients to develop selfdirected goals for change, developing the action plan for goal attainment, and proactively resolving the barriers in implementing the actions. ${ }^{15}$ The positive effects of this approach in enabling the patients with chronic conditions to translate the learned skills to day-to-day illness coping has been widely reported. ${ }^{16}$ Integrating this approach to strength-based dyadic intervention for PwMCI may promote more successful behavior changes that favor disease adaptation, as it allows carefully attending to their abilities, interests, values, preferences, and psychosocial contexts in the goal attainment process. ${ }^{15,16}$

Up to now, the strength-based concept has not been applied to promote effective coping with MCI in a dyadic fashion. The aim of this study was to fill this research gap and to examine the effect of a dyadic strength-based empowerment program (D-StEP-MCI) on the health outcomes of the PwMCI and their family caregivers. The study hypothesized that, as compared with the PwMCI who received the usual care, those in the D-StEP-MCI would report: 1) greater improvement in cognitive function and subjective memory, 2) a greater reduction in cognitive and neuro-psychiatric symptoms, and 3) a greater reduction in depressive symptoms, upon completion of the program and at 3 months thereafter. Meanwhile, for CGs who have received the D-StEP-MCI would report: 1) less stressful in managing the cognitive and neuro-psychiatric symptoms, 2) less dyadic relationship strain, and 3) a greater reduction in depressive symptoms at the same evaluative endpoints as the PwMCI.

\section{Materials and methods}

\section{Participants and setting}

This was a randomized controlled trial (RCT) with repeated post-test data collection. The Joint Chinese University of Hong Kong-New Territories East Clinical Research Ethics Committee has approved the study. The trial was registered at Center for Clinical Research and Biostatistics Trial Registry (CUHK-CCRB00604) and complied with the Declaration of Helsinki. The individual deidentified participant data 
including the demographic and cognitive characteristics will be available for 10 years from the date of publication and can be accessed by sending request to the corresponding author. The participants were recruited from an elderly community center in Hong Kong. The recruitment of the PwMCI-caregiver dyads was done in two steps. First, the research assistant identified the eligible PwMCI. According to the Consortium on Alzheimer's Disease Working Group, ${ }^{17}$ the criteria to define MCI including self-report of reduced cognitive function in the past year, clinical evaluation of cognitive decline as evidenced by a score of 18-22 on the Montreal Cognitive Assessment (Chinese version, MoCA-C), without a diagnosis of dementia and no major functional limitation in daily activities. With adjusting the influence of education level on cognitive performance by adding 1 point to the total score for those with less than 6 years of education, the MoCA-C gives the best sensitivity $(0.828 \%)$ and specificity $(0.735 \%)$ to identify PwMCI. ${ }^{18}$ The other selection criteria included: 1) able to identify one co-residing family caregiver to participate, 2) communicable with research team, 3) no pre-existing acute psychotic disease, and 4) consented to participate. Second, the research assistant contacted the referred family caregivers through phone contact for eligibility screening. The same set of selection criteria for PwMCI, except for the cognitive eligibility, was applicable to the family caregivers. The research assistant explained the purpose and procedure to the eligible PwMCI-caregiver dyads and obtained the informed written consent from both the PwMCI and the family caregivers. The care dyads were informed of the voluntary nature of participation and their right to withdraw from the study at anytime.

The sample size for the RCT was estimated by using RMASS. ${ }^{19}$ With reference to the dyadic intervention which incorporated the strength-based and empowerment approaches of care, ${ }^{12,20}$ an effect size of 0.6 on the cognitive and/or emotional outcomes of PwMCI and their family caregivers was used for power analysis. Accordingly, a total of 50 care dyads per study group was needed to achieve $80 \%$ power $(\alpha=0.05)$ and allowed for a $10 \%$ attrition for a 3-month post-test outcome evaluation. ${ }^{19}$ Block randomization with a block size of 12 using the restricted shuffle approach was used to ensure an even distribution of participants among the two study arms throughout the study period. ${ }^{21}$ Randomization was carried out using computer-generated random codes in sealed opaque envelopes to assign the 'D-StEP-MCI' or "usual care" sequence in blocks of 12 using an allocation ratio of 1:1.

\section{Study interventions}

The dyadic strength-based empowerment program for $\mathrm{MCl}$ (D-StEP-MCl)

The D-StEP-MCI is underpinned by the Roy Adaptation Model $^{22}$ and the Zimmer's Theory of Psychological Empowerment. ${ }^{23}$ Based on the Roy Adaptation Model, PwMCI is regarded as an adaptive system who need to adapt to the challenges brought by MCI. This requires increasing their coping capacities to optimize their adaption in the physical, self-concept, interdependence and role function domains. The empowerment theory emphasis such adaption can be promoted in a goal attainment process during which efforts are placed on increasing one's perceived self-control, optimize their comprehensibility of the situation, and create an environment which is compatible to their needs.

By integrating the strength-based and empowerment approach, the 14-week D-StEP-MCI focused at: a) motivating active participation of the care dyads, $b$ ) identifying their individual and collective strengths in coping with the cognitive decline, c) building upon effective self-care or caregiving situations as reported by the care dyads, and d) facilitating the care dyads to acquire new skills to adapt to the functional loss due to MCI. The D-StEP-MCI was delivered by the interventionist with a Bachelor degree in gerontological care. She received a 40-hr training from the investigation team on management of cognitive decline and the caregiving, health counseling techniques, empowermentbased goal-directed behavioral changes, and strength-based approach of care. The overall D-StEP-MCI consisted of three phases with details outlined in Figure 1.

Phase I: working out strength-based biographies for the care dyads (Ist-2nd week)

It was a 90-min dyadic session to assess five core areas of strengths including: 1) the life profile and preference of the dyads (such as vocational history, hobbies, normal daily routines, and preferences, dreams, etc.), 2) the level of functional performance of the PwMCI, 3) the effective and challenging caregiving situations and the handling strategies, 4) environmental and social care resources for designing home-based activities (e.g., availability of Wi-Fi, iPad, and social network system), and 5) the collective strengths of the dyads which might benefit disease adaptation such as good pre-morbid relationship, positive family values on caregiving, etc. A semi-structured interviewing guide was developed to serve the purpose. A biography was developed according to the assessment findings, and the interventionist went through its 


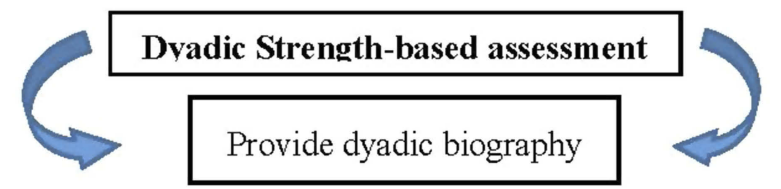

Workshop for PwMCI

Week 1: Handling memory loss

- Spaced retrieval;

- Use ABLED cues ;

- Memory compensation skills;

- Grouping techniques;

- External memory aids;

- Use long-term memory to engage in challenges task

\section{Dvadic workshop}

Week 2: Gauze the capability - Mirroring oneself identify 'important and meaningful activities;

identify frustration in its completion set a precise roadmap

Fitting a meaningful life schedule

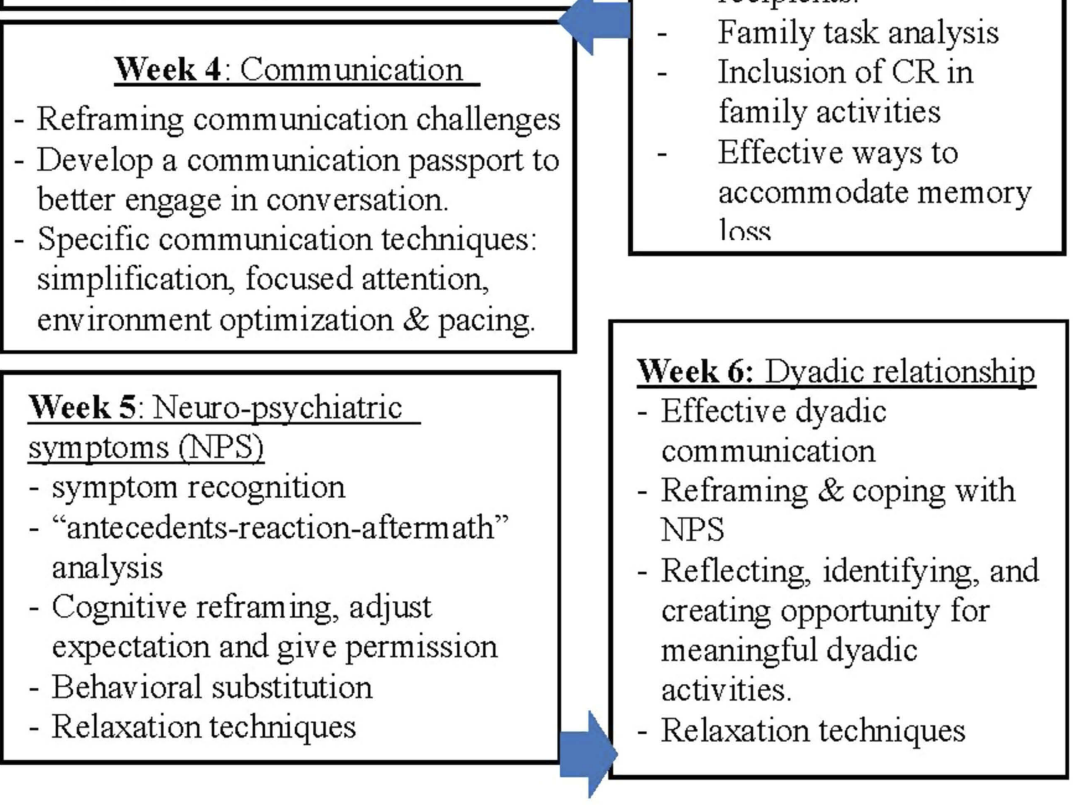

Figure I The dyadic strength-based empowerment program (D-StEP-MCI).

content with each care dyad at 1 week thereafter, with the purpose to increase the self-awareness and recognition of their own strengths. Photos provided by the dyads were inserted as appropriate to illustrate their individual strengths and dyadic relationship. The PwMCI was told to bring the biography to the subsequent empowering session in the next phase, so that the strengths at dyadic level could be integrated to the action plan developed to improving disease adaptation in the next Phase.
Phase 2: delivering strength-based empowering sessions (3rd-8th week)

The purpose of the strength-based empowering sessions is to optimize three core areas including (a) independence, (b) activity and social engagement, and (c) stress adaptation of the PwMCI-caregiver dyads. The sessions capitalized the abilities of the PwMCI-caregiver dyads as identified in Phase 1 and empowered them to acquire new skills in living with the cognitive, emotional, and 
behavioral changes. The six weekly sessions are educative-counseling in nature, with four of them delivered to the PwMCI in a small group of six in the community center. The other two sessions were delivered to the care dyads at their home. The sessions to the caregivers were scheduled at the week after every two consecutive sessions for the PwMCI.

For the session to the PwMCI, it covered four topics including: 1) managing memory loss, 2) staying active: gauge the capability, 3) communication passport, and 4) handling emotional and behavioral problems. Figure 1 outlines the focus of each session. Each session was commenced with a discussion about the impact of MCI on everyday life regarding the topic area of concern, what currently worked for the PwMCI (strengths) and what problems encountered. Then, the interventionist introduced various strategies to cope with the problems relevant to the topic area. Table 1 outlines the main purpose and specific skills of each session. Interactive role-play, demonstration and return demonstration were used to enable the participants to integrate the new skills and optimize their existing strength for their life situation. Thereafter, a goal-setting process was initiated to help the participants to set the person-centered goals related to the identified care needs. An action plan was formulated accordingly, with emphasis placed on optimizing the use of the individual and collective strengths of the care dyads as identified in their own biography. The level of goal attainment was monitored in the subsequent sessions and additional advice for resolving the barriers was brainstormed in the group. The goals and action plans were documented in the last session of the biography.

The topics of home-based sessions for the care dyads echoed with the previous two sessions provided to the PwMCI. The first session was titled as "dyadic approach to optimize family inclusion" which focused on strategies to enable effective management of memory deficit and optimize the opportunities of PwMCI for role and social engagement. The second session was "strengthening dyadic relationship" through effective dyadic communication and interaction in the presence of cognitive deficit and neuro-psychiatric symptoms. The aims of these homebased session not only focused on supporting the PwMCI to better translate the strategies covered in the group session to their individual home settings, but, more importantly, empowered the family caregivers and solicited their support to provide a facilitating social and actual environment favored such changes. Additional actions from the dyadic perspective for the goals set in the previous groupbased sessions were entered to the biography.

\section{Phase 3: telephone follow-up (9th-14th week)}

Upon the completion of the six empowerment sessions, three bi-weekly telephone calls were made to the PwMCI and family caregivers to monitor the level of goal attainment. They were encouraged to use the speakerphone to engage in the conversation if both of them were physically together. Additional support and advices were given accordingly. The interventionist also discussed with the dyads about any newly evolved challenges for maintaining independence, engagement, and stress adaptation.

\section{Control intervention}

The control intervention included the usual care provided by the elderly community. Basically, there were monthly meeting to disseminate the entertaining and health promotion activities for the members including older adults and their family caregivers. In the study period, no activities using strength-based or empowerment-based concept were organized. There was also no health promotion activities specifically designed for PwMCI and their family caregivers.

\section{Outcome evaluation}

The effects of the D-StEP-MCI were evaluated at three endpoints, including baseline (T0), immediately after the 14-week strength-based dyadic program (T1), and at 3 months thereafter (T2). For the PwMCI, the evaluative outcomes include cognitive function, subjective memory, neuro-psychiatric symptoms, and depressed mood. As for the caregivers, the burden associated with symptom management and depressed mood was evaluated. Data collection with the following instruments were collected at the community center or at the dyad's home.

The Mini-Mental State Examination (Cantonese version; MMSE) was used to assess the cognitive function. It covers five crucial cognitive functions including registration, attention and calculation, recall, language, ability to follow a simple command, and orientation. The Cronbach's alpha was reported as 0.86 when used in the Hong Kong population. $^{24}$

The 24-item Memory Inventory for Chinese (MIC) was used to assess the subjective memory of the PwMCI. It consists of 24 items asking the respondents' awareness of their own memory deficits. The response set is dichotomous, with higher score represents more severe subjective 
memory loss. The MIC was found to be reliable and valid when used in the Chinese population. ${ }^{25}$

The 24-item Revised Memory and Behavioural Problem Checklist was used to assess the memory and neuro-psychiatric symptoms of the participants with MCI and their caregivers' burden in managing such symptoms. ${ }^{26}$ The caregivers reported the presence of symptom on a dichotomous scale and the level of perceived disturbance for the identified symptom on a ' $0-4$ ' Likert scale, with higher scores indicating more severe symptom severity and higher caregiver stress. The Cronbach's alpha was 0.82-0.90 and its construct validity was evident by its significant association with depression in the Chinese population. ${ }^{26}$

The 10-item Center for Epidemiologic Studies Depression Scale (C-ESD-10, Chinese version) was used to measure depressive mood in the care dyads. ${ }^{27}$ The response set is a 4point Likert scale with a higher score indicating a higher level of depression. The Cronbach's alpha of CES-D was 0.78 with good convergent validity with the DSM-III-R when used in the Chinese population. ${ }^{28}$

\section{Data analysis}

Data were double-entered for validation. Descriptive statistics was used to summarize the characteristics of the participants. The Skewness and Kurtosis statistics were used to evaluate the normality of all the continuous outcome variables. Between-group comparisons at baseline were made using $t$-test, Mann-Whitney test, Pearson Chi-square, or Fisher's exact test using SPSS 22.0, as appropriate, with $p<0.025$ implying the need for adjustment. Generalizing Estimating Equation was used to compare the differential changes on the outcomes between intervention and control groups across three endpoints including (T0, T1, and T2). ${ }^{29}$ This method has the merits of accounting for intra-correlated pretest-post-test and accommodating missing data and time-varying covariate. ${ }^{30}$ The level of significance was set at 0.05 (two-sided).

\section{Results}

Figure 2 shows the CONSORT flow diagram for the PwMCI-caregiver dyads. From February 2018 to January 2019, the research assistant conducted cognitive and eligibility screening to 304 members of the elderly community center using the MoCA. Among which, 138 people with MCI met the eligibility criteria. Of these, 103 care dyads finally agreed to participate. Block randomization allocated 52 of them to receive the D-StEP-MCI and the other 51 received the usual care. For the participants with MCI, 4 of them dropped out before T1 and anther 10 dropped out by $\mathrm{T} 2$. As for the caregivers, 7 dropped out by $\mathrm{T} 1$ and another 11 by $\mathrm{T} 2$. The reasons for dropout included not interested to complete the post-test measure, lack of time availability, left the town, hospitalization, and loss of contact. There was no significant difference in the demographic characteristics and/or MMSE scores between PwMCI and the caregivers who completed and withdrawn from the study.

\section{Characteristics of the care dyads}

Table 1 outlines the details of the characteristics of the care dyads. The mean age of the care recipients was 80.2 $(\mathrm{SD}=7.3)$ and over $70 \%$ of them were female. The education level was fair and over $70 \%$ of them received only primary level education or below. The MoCA score ranged from 18 to 22, with the mean as 19.77 ( $\mathrm{SD}=1.22)$. As for the family caregivers, the mean age was $58.9(\mathrm{SD}=15.2)$ and female was also over-represented. Majority of them were the children or in-law of the care recipients. There was no significant difference in the characteristics and baseline outcome variables between the participants who received the strength-based dyadic program and usual care.

\section{Effects on health outcomes of $\mathrm{MCl}$ participants}

The baseline, post-test, and the 3-month health outcome evaluation of the MCI participants are listed in Table 2. For cognitive function, the PwMCI in the D-StEP-MCI group had a significantly greater improvement in the MMSE score compared to the usual care group, across the baseline and post-test period $(\beta=-1.79, \mathrm{SE}=0.69$, $p<0.001$ ), but such improvement cannot be sustained at the 3-month endpoint. The D-StEP-MCI also improved the subjective memory of the PwMCI as they reported a reduced MIC score over the study period (i.e., improved subjective memory) whereas the usual care group had deterioration on that perceived function. GEE results indicated there were significant between-group differences in the change of the MIC score over the baseline and posttest period $(\beta=7.54, \mathrm{SE}=2.79, p=0.007)$, and such difference was maintained up to the 3 -month post-test endpoint $(\beta=8.16, \mathrm{SE}=3.13, p=0.009)$. Similar positive program effects were found in the memory and behavioral symptoms and the D-StEP-MCI group showed a significant reduction in symptom severity than the usual care group over the two evaluative endpoints (post-test: $\beta=2.90$, 


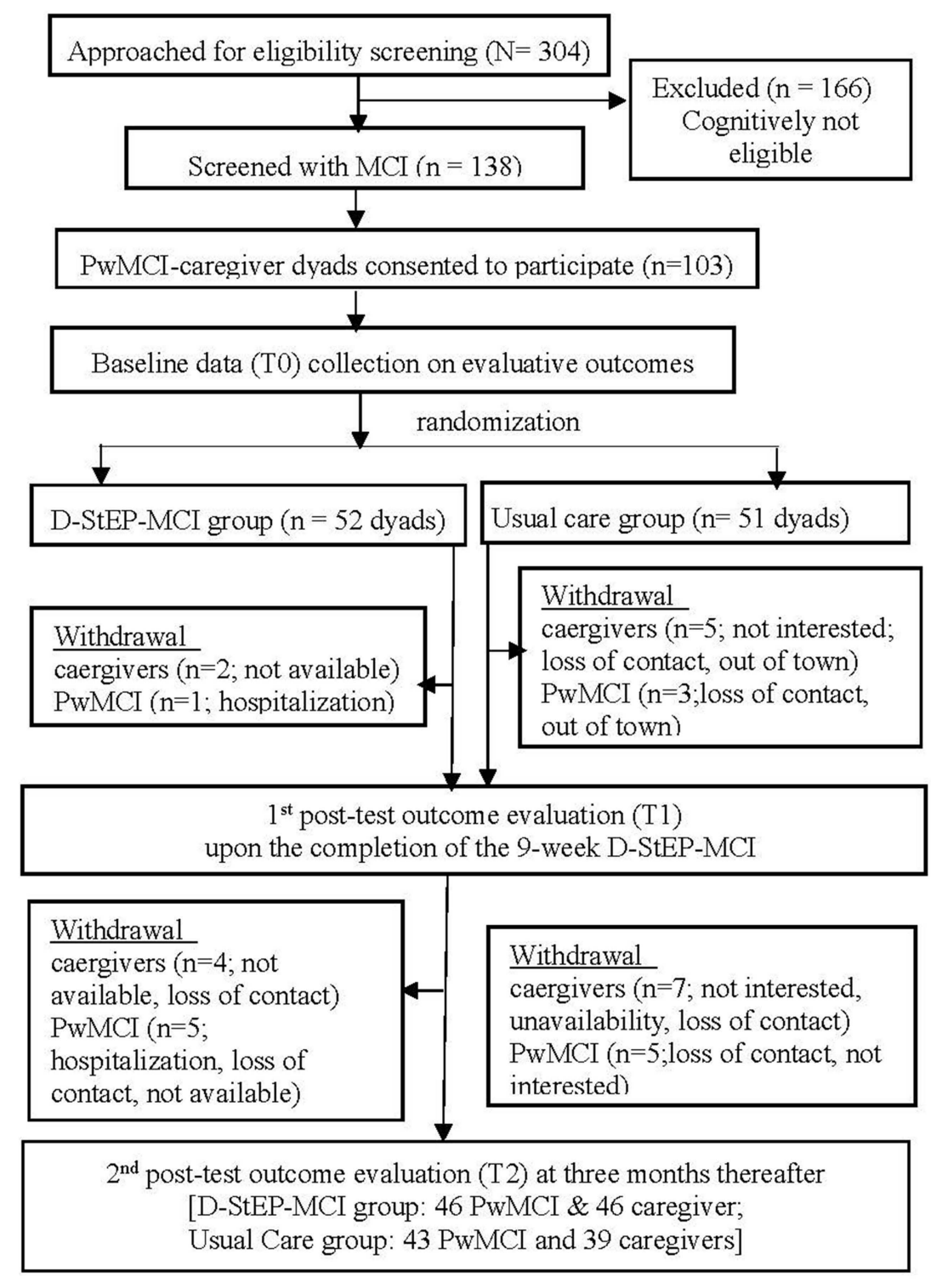

PwMCI $=$ Persons with mild cognitive impairment; D-StEP-MCI $=$ Dyadic Strength-based Empowerment Program

Figure $\mathbf{2}$ The consolidated standards of reporting trial diagram.

$\mathrm{SE}=0.84, p=0.001 ; 3$-month post-test: $\beta=3.01, \mathrm{SE}=1.11$, $p=0.007)$. The MCI participants in the D-StEP-MCI group also reported a greater improvement in depressive mood than those in the usual care group at the two evaluative endpoints (post-test: $\beta=2.67, \mathrm{SE}=1.12, p=0.017 ; 3$ month post-test: $\beta=3.57, \mathrm{SE}=1.31, p=0.006$ ).

\section{Effects on the caregivers' health outcomes}

Table 2 also lists the results of the effects of D-StEPMCI on the caregivers' health outcomes. Echoed with the reduction in the memory and behavioral symptoms of the MCI participants, the caregivers in the D-StEPMCI group reported a greater reduction in the disturbance associated with such symptoms over the period from baseline to the post-test $(\beta=3.29, \mathrm{SE}=1.50$, $p=0.028$ ) but not the 3 -month endpoint. On the other hand, family caregivers in the SDP group reported a significant greater reduction in depressed mood than the usual care group from the baseline to the longer evaluative endpoints $(\beta=372, \mathrm{SE}=1.51, p=0.014)$. 
Table I Characteristics of the participants with $\mathrm{MCl}$ and their family caregivers

\begin{tabular}{|c|c|c|c|}
\hline & Strength-based dyadic program $(n=52)$ & Usual care $(n=51)$ & p-value \\
\hline \multicolumn{4}{|l|}{ Participants with $\mathrm{MCl}$} \\
\hline Age & $81.0 \pm 7.0$ & $79.8 \pm 7.4$ & 0.38 \\
\hline Female gender & $40(76.9 \%)$ & $43(84.3 \%)$ & 0.343 \\
\hline Education level & & & 0.712 \\
\hline No formal education & $16(30.8 \%)$ & $\mathrm{II}(2 \mathrm{I} .6 \%)$ & \\
\hline Primary level & $24(46.2 \%)$ & $26(51.0 \%)$ & \\
\hline Secondary level & $\mathrm{II}(2 \mathrm{I} .2 \%)$ & $12(23.5 \%)$ & \\
\hline Tertiary level & $\mathrm{I}(1.9 \%)$ & $2(3.9 \%)$ & \\
\hline Married status & & & 0.125 \\
\hline Single & $0(0 \%)$ & $3(6.0 \%)$ & \\
\hline Married & $22(42.3 \%)$ & $24(47.1 \%)$ & \\
\hline Divorced & $\mathrm{I}(\mathrm{I} .9 \%)$ & $2(3.92 \%)$ & \\
\hline Widowed & $29(55.8 \%)$ & $22(43.1 \%)$ & \\
\hline MMSE & $23.12 \pm 3.71$ & $23.8 I \pm 3.72$ & 0.348 \\
\hline RMBPC & $6.60 \pm 4.43$ & $5.67 \pm 5.31$ & 0.663 \\
\hline MIC & $20.02 \pm 10.33$ & $15.14 \pm 9.76$ & $0.08 I$ \\
\hline CES-D & $6.33 \pm 4.12$ & $6.14 \pm 4.02$ & 0.884 \\
\hline \multicolumn{4}{|l|}{ Family caregivers } \\
\hline Age & $59.8 \pm 15.2$ & $59.5 \pm 16.7$ & 0.93 \\
\hline Gender & 39 (75.0\%) & 32 (62.7\%) & 0.062 \\
\hline Education level & & & 0.675 \\
\hline No formal education & $3(5.8 \%)$ & I (2.0\%) & \\
\hline Primary level & 9 (17.3\%) & 7 (I3.7\%) & \\
\hline Secondary level & $26(50 \%)$ & $26(51.0 \%)$ & \\
\hline Tertiary level & 14 (26.9\%) & 17 (33.3\%) & \\
\hline Marital status & & & 0.591 \\
\hline Single & $15(28.8 \%)$ & II (2I.6\%) & \\
\hline Married & 34 (65.4\%) & $34(66.7 \%)$ & \\
\hline Divorced & I (I.9\%) & $3(5.9 \%)$ & \\
\hline Widowed & $2(3.8 \%)$ & $3(5.9 \%)$ & \\
\hline Occupation & & & 0.668 \\
\hline Full-time & $20(38.5 \%)$ & 19 (37.3\%) & \\
\hline Part-time & 6 (11.5\%) & $3(5.9 \%)$ & \\
\hline Not working & $26(50 \%)$ & $29(56.9 \%)$ & \\
\hline Relationship with care recipients & & & 0.453 \\
\hline Spouse & $18(34.6 \%)$ & $14(27.5 \%)$ & \\
\hline Children/children in-law & $29(55.8 \%)$ & $34(66.7 \%)$ & \\
\hline Grand children & $3(5.8 \%)$ & $3(5.9 \%)$ & \\
\hline Sibling & $2(3.8 \%)$ & $0(0 \%)$ & \\
\hline RMBPS-Response & $6.27 \pm 4.28$ & $6.22 \pm 4.21$ & 0.947 \\
\hline CES-D & $6.25 \pm 3.54$ & $6.10 \pm 3.13$ & 0.886 \\
\hline
\end{tabular}

Abbreviations: $\mathrm{MCl}$, mild cognitive impairment; MMSE, mini-mental state examination; RMBPC, revised memory and behavioral problems checklist; MIC, Memory Inventory for Chinese; CES-D, Center for Epidemiologic Study - Depression Scale.

\section{Discussion}

This is the first study to integrate both strength-based and empowerment approach to a dyadic intervention (D-StEPMCI) for improving the health outcomes of PwMCI and their family caregivers. The results are very encouraging as the D-StEP-MCI not only improved the cognitive and neuro-psychiatric symptoms of PwMCI but also reduced the depression for the care dyads. A positive program effect on the caregiver stress associated with MCI symptom management is also noted. By firstly identifying and communicating the preserved strengths in a dyadic fashion, and then empowering the PwMCI-caregiver dyads to 
Table 2 Changes in the health outcomes of the participants with $\mathrm{MCl}$ and their family caregivers

\begin{tabular}{|c|c|c|c|c|c|c|c|c|}
\hline \multirow[t]{2}{*}{ Outcomes } & \multirow{2}{*}{$\begin{array}{l}\text { Strength-based } \\
\text { dyadic program }\end{array}$} & \multirow{2}{*}{$\begin{array}{l}\text { Usual } \\
\text { care }\end{array}$} & \multicolumn{2}{|l|}{ Time effect } & \multicolumn{2}{|l|}{ Group effect } & \multicolumn{2}{|c|}{ Time $\times$ Group effect } \\
\hline & & & Beta (SE) & $p$-value & Beta $(95 \% \mathrm{Cl})$ & $p$-value & Beta $(95 \% \mathrm{Cl})$ & $p$-value \\
\hline \multicolumn{9}{|c|}{ Outcomes of participants with $\mathrm{MCl}$} \\
\hline MMSE & & & & & $0.65(0.74)$ & 0.38 & & \\
\hline Baseline & $23.12 \pm 3.7 \mid$ & $23.81 \pm 3.72$ & & & & & & \\
\hline Post-test & $25.81 \pm 3.35$ & $24.67 \pm 3.54$ & $2.73(0.48)$ & $<0.001$ & & & $-1.79(0.69)$ & 0.010 \\
\hline 3-month & $24.63 \pm 3.68$ & $24.07 \pm 4.08$ & $1.48(0.57)$ & 0.009 & & & $-1.26(0.74)$ & 0.088 \\
\hline MIC & & & & & $-5.213(2.93)$ & 0.066 & & \\
\hline Baseline & $20.02 \pm 10.33$ & $15.14 \pm 9.76$ & & & & & & \\
\hline Post-test & $|4.59 \pm 8.5|$ & $16.92 \pm 7.76$ & $-5.66(2.11)$ & 0.007 & & & $7.54(2.79)$ & 0.007 \\
\hline 3-month & $|5.35 \pm 8.5|$ & $17.68 \pm 8.16$ & $-5.36(2.30)$ & 0.020 & & & $8.16(3.13)$ & 0.009 \\
\hline RMBPC & & & & & $-0.83(0.98)$ & 0.399 & & \\
\hline Baseline & $6.60 \pm 4.43$ & $5.67 \pm 5.31$ & & & & & & \\
\hline Post-test & $4.10 \pm 3.12$ & $6.09 \pm 4.41$ & $-2.58(0.63)$ & $<0.001$ & & & $2.90(0.84)$ & 0.001 \\
\hline 3-month & $4.76 \pm 3.15$ & $8.85 \pm 6.17$ & $-1.80(0.66)$ & 0.006 & & & $3.02(1.11)$ & 0.007 \\
\hline CES-D & & & & & $-0.58(1.33)$ & 0.966 & & \\
\hline Baseline & $6.33 \pm 4.12$ & $6.14 \pm 4.02$ & & & & & & \\
\hline Post-test & $5.73 \pm 3.92$ & $8.33 \pm 6.13$ & $-0.69(0.86)$ & 0.424 & & & $2.67(1.12)$ & 0.017 \\
\hline 3-month & $4.48 \pm 3.24$ & $7.91 \pm 6.87$ & $-1.94(0.85)$ & 0.023 & & & $3.57(1.31)$ & 0.006 \\
\hline \multicolumn{9}{|c|}{ Caregivers' outcomes } \\
\hline RMBPC-R & & & & & $-0.020(1.72)$ & 0.991 & & \\
\hline Baseline & $6.27 \pm 4.28$ & $6.22 \pm 4.21$ & & & & & & \\
\hline Post-test & $3.98 \pm 5.18$ & $7.11 \pm 6.43$ & $-2.48(0.90)$ & 0.006 & & & $3.29(1.50)$ & 0.028 \\
\hline 3-month & $4.76 \pm 7.00$ & $8.85 \pm 7.34$ & $-1.94(0.99)$ & 0.051 & & & $4.05(2.34)$ & 0.083 \\
\hline CES-D & & & & & $-0.084(1.09)$ & 0.94 & & \\
\hline Baseline & $6.25 \pm 3.54$ & $6.10 \pm 3.13$ & & & & & & \\
\hline Post-test & $5.65 \pm 4.78$ & $5.70 \pm 4.52$ & $-0.54(0.67)$ & 0.419 & & & $0.209(1.04)$ & 0.084 \\
\hline 3-month & $4.48 \pm 4.34$ & $7.91 \pm 6.89$ & $-1.85(0.99)$ & 0.062 & & & $3.72(\mathrm{I} .5 \mathrm{I})$ & 0.014 \\
\hline
\end{tabular}

Abbreviations: $\mathrm{MCl}$, mild cognitive impairment; MMSE, mini-mental state examination; RMBPC, revised memory and behavioral problems checklist; MIC, Memory Inventory for Chinese; CES-D, Center for Epidemiologic Study - Depression Scale.

mobilize the navigated capabilities to cope with the changes associated with MCI, a better disease adaptation at the personal and dyadic level was resulted.

The study results converge with the findings of a review on dyadic interventions for persons with dementia (PwD). ${ }^{7}$ Even though such dyadic interventions covered a wide range of care approaches including support group, health counseling, cognitive stimulation, compensatory methods, and skill training, the results consistently show the merit of dyadic interventions in improving the cognitive function of PwD and the psychosocial adjustment between the care dyads. The reason may be related to the fact that coping with cognitive dysfunction is a challenging life experience, and the mutual influence between the care dyads may shape the adaptation process. ${ }^{31}$ By providing the intervention in a dyadic fashion, their collaborative effort for disease coping can be drawn by which misunderstanding and miscommunication between the dyads would be minimized. Among the study in this review, one study also adopted a strength-based approach by which cognitive rehabilitation and counseling-based training were combined to provide the care dyads with six home-based sessions on similar topics as the current study. ${ }^{13}$ Emphasis was placed on supporting the care dyads to translate a set of core skills relevant to their own situation in day-to-day life. Consistent with our study, the strength-based dyadic intervention significantly improved the psychosocial adaptation of the family caregivers. The positive effect may be related to the fact that their active participation in the intervention provided them with more insights on how to interact and engage the persons with cognitive deficit in a more constructive manner, and thereby smoothed out the adaptation process.

As compared with another pilot study, which applied strength-based dyadic approach to engage the PwMCI in daily meaningful activities, ${ }^{5}$ our study showed more 
promising effects of the D-StEP-MCI in improving the mood status of the care dyads as well as the cognitive and neuro-psychiatric symptom management of the PwMCI. Although the inconsistent findings may be related to the lack of statistical power in the prior pilot study, several unique features of the D-StEP-MCI may also explain for its more favorable effects.

Firstly, although both of the above-mentioned strengthbased interventions supported the PwMCI-caretaker dyads to navigate for the remaining capabilities, the good qualities in the dyadic relationship and the successful caregiving experience, ${ }^{5}$ the current study (D-StEP-MCI) took an additional step to integrate the identified strengths to a written biography. Going through the biography with the care dyads served the function of enabling them to refocus and reflect on their strengths and the previous successful experience in coping with the cognitive, emotional, and interactional problems associated with MCI. Such reflective practice was found to be very effective in promoting a positive outlook on oneself ${ }^{32}$ and may eventually lead to a stan improved mood status and better motive and higher confidence for the disease adaptation.

Second, the D-StEP-MCI placed greater emphasis on promoting effective disease adaptation from a social interactional perspective. In particular, whereas there were four group-based sessions to empower the PwMCI to optimize the use of cognitive strength, enhance role participations, overcome communication deficit, and increase emotional regulation, two dyadic sessions were scheduled between these group-based sessions to empower the family caregiver to build a more favorable social and interactional context for the PwMCI. Such design was based on the premise that effective disease adaptation for MCI required a social context that facilitates role and social engagement even in the presence of cognitive affliction. ${ }^{33}$ Indeed, interpersonal difficulties have been consistently found to trigger more neuro-psychiatric symptoms. ${ }^{34}$ The dyadic session which focused on how to enhance the dyadic communication and interaction may attribute to the positive effect of D-StEP-MCI in the corresponding symptom control, and resulted in better psychological adjustment.

Lastly, integrating an empowerment approach to the DStEP-MCI may also attribute to its more favorable longerterm effects at the three-month evaluative endpoint. This is because the empowerment strategies placed emphasis on facilitating the PwMCI and their caregivers to translate the taught subject matter to daily practice. "Self-determination" is central to the overall empowerment process, and the intervener acted as an enabler to facilitate them to set their own goals for disease adaptation in a dyadic fashion. Substantial evidence has indicated that such a client-centered goal-setting approach is effective to increase one's sense of control and reduce the level of uncertainty in the disease adaptation process. ${ }^{16}$ Together with delivering the dyadic sessions at the home environment, the translation of the behavioral changes to real-life environment could be further facilitated. As such, the empowerment approach may potentiate the effects of strength-based intervention by enabling the care dyads actively integrate the navigate capabilities in the actual disease coping process.

Nevertheless, the D-StEP-MCI did not demonstrate a longer-term effect on objective cognitive function. This may be related to the fact that the model relies on the enhanced role and social engagement of the PwMCI in daily life to influence the cognitive outcomes, and no active and structured cognitive training component was incorporated. Such program design, together with the progressive deteriorating nature of MCI, may explain for the absence of longer-term cognitive benefit. Future research may examine the effects of an enhanced version by incorporating a stronger cognitive training component to the home-based practice. In addition, the feasibility of applying the model to a more extended family rather than dyadic context can be explored. This is especially true, as the more capable PwMCI may not rely on a single family member as the primary caregivers. The process of disease adaptation is, indeed, evolved from a family context. Finally, as persons with mild dementia also has certain level of preserved performance function, future research can explore the feasibility and effects of extending the D-StEP to this clinical cohort.

This study provides important insights into the development of disease management model for MCI. The findings suggested the value of incorporating strength-based and empowerment approaches to the disease management model for MCI. Instead of just focusing on compensating the functional deficit (e.g., cognitive training), providing more comprehensive care in a dyadic fashion is strongly indicated. Guided self-reflection can be used to support the care dyads to identify and focus on the existing strengths, such as the functional performance of the PwMCI, positive caregiving experience and relational qualities, so that the dyads would perceive a higher level of confidence and selfcontrol in the disease adaptation process. Using a partnership and goal-setting approach further provides the dyads with an interactive roadmap about how to integrate the 
identified strengths to optimize their role participation. Indeed, family caregiver involvement is particularly important in supporting the PwMCI, as family provides the immediate social context to allow a higher level of independence and active social engagement. As MCI is an intermediate prodromal preclinical stage of dementia, the above strategies not only promote effective adaptation of the care dyads during the critical window period, but also prepare them for more active dyadic coping when cognitive decline develops to a more undesirable stage.

The study is not without limitation. Firstly, over $70 \%$ of the PwMCI were female as the sample was recruited from the elderly community center where they had more female than male older adults. The generalizability of the findings to the male older adults needs to be cautious. Secondly, their attrition rate for the control group was above the estimation (10\%), and the situation was even worse for the family caregivers as almost $40 \%$ of them were working full time. The lack of attentional placebo may reduce their motivation to participate in the posttest data collection. Nevertheless, there was no significant difference in the demographic background among those who complete or dropout from the study. Providing motivational incentives may be a strategy to reduce the attrition.

\section{Conclusion}

MCI is a challenging experience during which the persons with this condition and the family caregivers have to adjust to cognitive, behavioral, and emotional disease manifestation. This study demonstrated the health benefits of supporting the PwMCI and caregiver dyads to navigate the preserved strengths and resources for incorporation to the disease coping. The findings have also underscored the importance of using social interactional perspective in promoting adaption to MCI at a dyadic fashion.

\section{Disclosure}

The authors report no conflicts of interest in this work.

\section{References}

1. Prince M, Comas-Herrera A, Knapp M, Guerchet M, Karagiannidou M. World Alzheimer report 2016: improving healthcare for people living with dementia: coverage, quality and costs now and in the future. Available from: http://eprints.lse.ac.uk/id/eprint/67858 Accessed September 26, 2016.

2. Wadley VG, Unverzagt FW, McGuire L, et al. Incident cognitive impairment is elevated in the stroke belt: the REGARDS study. Ann Neurol. 2011;70(2):229-236. doi:10.1002/ana.22432

3. Sachdev PS, Lipnicki DM, Kochan NA, et al. The prevalence of mild cognitive impairment in diverse geographical and ethnocultural regions: the COSMIC collaboration. PLoS One. 2015;10(11):1-19. doi:10.1371/journal.pone. 0142388
4. Petersen RC, Smith GE, Waring SC, Ivnik RJ, Tangalos EG, Kokmen E. Mild cognitive impairment: clinical characterization and outcome. Arch Neurol. 1999;56(3):303-308. doi:10.1001/archneur.56.3.303

5. Dean KJ, Jenkinson C, Wilcock GK. Experiences of healthcare services in people with mild cognitive impairment and their carers. Age Ageing. 2013;42(suppl 3):iii27-8.

6. Lu YFY, Haase JE. Experience and perspectives of caregivers of spouse with mild cognitive impairment. Curr Alzheimer Res. 2009;6(4):384-391.

7. Domingues NS, Verreault P, Hudon C. Reducing burden for caregivers of older adults with mild cognitive impairment: a systematic review. Am J Alzheimers Dis Other Demen. 2018;33(7):401-414. doi: $10.1177 / 1533317518788151$

8. Salthouse TA. Mental exercise and mental aging: evaluating the validity of the "use it or lose it" hypothesis. Perspect Psychol Sci. 2006;1(1):68-87. doi:10.1111/j.1745-6916.2006.00005.x

9. Banningh LW, Vernooij-Dassen MJ, Vullings M, Prins JB, Rikkert MG, Kessels RP. Learning to live with a loved one with mild cognitive impairment: effectiveness of a waiting list controlled trial of a group intervention on significant others' sense of competence and well-being. Am J Alzheimers Dis Other Demen. 2013;28(3):228238. doi: $10.1177 / 1533317513481093$

10. Lu YY, Bakas T, Yang Z, Weaver MT, Austrom MG, Haase JE. Feasibility and effect sizes of the revised daily engagement of meaningful activities intervention for individuals with mild cognitive impairment and their caregivers. J Gerontol Nurs. 2016;42(3):4558. doi:10.3928/00989134-20160212-08

11. Schmitter-Edgecombe M, Dyck DG. Cognitive rehabilitation multifamily group intervention for individuals with mild cognitive impairment and their care-partners. J Int Neuropsychol Soc. 2014;20 (9):897-908. doi:10.1017/S1355617714000782

12. Moon H, Adams KB. The effectiveness of dyadic interventions for people with dementia and their caregivers. Dementia. 2013;12 (6):821-839. doi:10.1177/1471301212447026

13. Judge KS, Yarry SJ, Looman WJ, Bass DM. Improved strain and psychosocial outcomes for caregivers of individuals with dementia: findings from project ANSWERS. Gerontologist. 2012;53(2):280292. doi:10.1093/geront/gns076

14. Blieszner R, Roberto KA. Care partner responses to the onset of mild cognitive impairment. Gerontologist. 2009;50(1):11-22. doi:10.1093/geront/gnp068

15. Anderson RM, Funnell MM. Patient empowerment: myths and misconceptions. Patient Educ Couns. 2010;79(3):277-282. doi:10.1016/ j.pec.2009.07.025

16. Werbrouck A, Seinnen E, Kercof E, Buyl R, Beckwee D, De Wit L. How to empower patients? A systematic review and meta-analysis. Transl Behav Med. 2018;8(5):660-674. doi:10.1093/tbm/iby064

17. Portet F, Ousset PJ, Visser PJ, et al. Mild cognitive impairment (MCI) in medical practice: a critical review of the concept and new diagnostic procedure. Report of the MCI Working Group of the European Consortium on Alzhemier's Disease. J Neurol Neurosurg Psychiatry. 2006;77(6):714-718. doi:10.1136/jnnp.2005.085332

18. Yeung PY, Wong LL, Chan CC, Leung JLM, Yung CY. A validation study of the Hong Kong version of Montreal Cognitive Assessment (HK-MoCA) in Chinese older adults in Hong Kong. Hong Kong Med J. 2014;20(6):504-510. doi:10.12809/hkmj144219

19. Hedeker D, Gibbons RD, Waternaux C. Sample size estimation for longitudinal designs with attrition: comparing time-related contrasts between two groups. J Educ Behav Stat. 1999;24:70-93. doi:10.3102/ 10769986024001070

20. Gitlin LN, Hodgson N, Jutkowitz E, Pizzi L. The cost-effectiveness of a nonpharmacologic intervention for individuals with dementia and family caregivers: the tailored activity program. Am J Geriatr Psychiatry. 2010;18:501-519. doi:10.1097/JGP.0b013e3181c37d13

21. Loehlin JC. Latent Variable Models: an Introduction to Factor, Path, and Structural Equation Analysis. Mahwah (NJ): Lawrence Erlbaum Associates; 2004. 
22. Roy C. Extending the Roy Adaption Model to meet changing global needs. Nurs Sci Q. 2011;24L:345-351. doi:10.1177/0894318411419210

23. Zimmerman MA. Psychological empowerment: issues and illustrations. Am J Commun Psychol. 1995;23:581-599. doi:10.1007/BF02506983

24. Chiu HFK, Lee HC, Chung WS, Kwong PK. Reliability and validity of the Cantonese version of the Mini-Mental State Examination: a preliminary study. East Asian Arch Psychiatry. 1994;SP2:25-28.

25. Lui VWC, Lam LCW, Chiu HFK. Validation of a memory inventory for the assessment of awareness of memory deficits in Alzheimer's disease in Chinese elderly. Int J Geriatr Psychiatry. 2006;21:917923. doi:10.1002/gps.1580

26. Fuh JL, Liu CY, Wang SJ, Wang HC, Liu HC. Revised memory and behaviour problems checklist in Taiwanese patients with Alzheimer's disease. Int Psychogeriatr. 1999;11:181-189.

27. Cheng ST, Chan ACM. The Center for Epidemiologic Studies Depression Scale in older Chinese: thresholds for long and short forms. Int $J$ Geriatr Psychiatry. 2005;20:465-470. doi:10.1002/ gps. 1314

28. Cheng ST, Chan ACM. Detecting depression in Chinese adults with mild dementia: findings with two versions of the Center for Epidemiologic Studies Depression Scale. Psychiatry Res. 2008;159:44-49. doi:10.1016/j.psychres.2007.06.023
29. Singer JD, Willett JB. Applied Longitudinal Data Analysis: Modelling Change and Event Occurrence. New York: Oxford University Press; 2003.

30. Handy JA, Negassa A, Forrester JE. Statistical analysis of correlated data using generalized estimating equations: an orientation. Am J Epidemiol. 2003;157(4):364-375. doi:10.1093/aje/kwf215

31. Roberto KA, McCann BR, Blieszner R. Trajectories of care: spouses coping with changes related to mild cognitive impairment. Dementia. 2011;12(1):45-62. doi:10.1177/1471301211421233

32. McKeown J, Clarke A, Ingleton C, Ryan T, Repper J. The use of life story work with people with dementia to enhance person-centred care. Int J Older People Nurs. 2010;5(2):148-158. doi:10.1111/ j.1748-3743.2010.00219.x

33. Preston L, Marshall A, Bucks RS. Investigating the ways that older people cope with dementia: a qualitative study. Aging Ment Health. 2007;11(2):131-143. doi:10.1080/13607860600844572

34. Feast A, Orrell M, Charlesworth G, Melunsky N, Poland F, Monizcook E. Behavioral and psychological symptoms in dementia and the challenges for family careers: systematic review. The Brit Jf Psychiat. 2016;208(5):429-434. doi:10.1192/bjp.bp.114.153684
Clinical Interventions in Aging

\section{Publish your work in this journal}

Clinical Interventions in Aging is an international, peer-reviewed journal focusing on evidence-based reports on the value or lack thereof of treatments intended to prevent or delay the onset of maladaptive correlates of aging in human beings. This journal is indexed on PubMed Central, MedLine, CAS, Scopus and the Elsevier
Dovepress

Bibliographic databases. The manuscript management system is completely online and includes a very quick and fair peer-review system, which is all easy to use. Visit http://www.dovepress.com/ testimonials.php to read real quotes from published authors. 